\title{
Strictly finite-range potential for light and heavy nuclei
}

\author{
P. Salamon, ${ }^{1}$ R. G. Lovas, ${ }^{1}$ R. M. Id Betan, ${ }^{2,3}$ T. Vertse,,${ }^{1,4}$ and L. Balkay ${ }^{5}$ \\ ${ }^{1}$ MTA Institute for Nuclear Research, Debrecen, P.O. Box 51, H-4001, Hungary \\ ${ }^{2}$ Department of Physics and Chemistry (FCEIA-UNR), Avenida Pellegrini 250, S2000BTP Rosario, Argentina \\ ${ }^{3}$ Physics Institute of Rosario (CONICET), Boulevard 27 de Febrero 210 bis, S2000FPA Rosario, Argentina \\ ${ }^{4}$ Faculty of Informatics, University of Debrecen, P.O. Box 12, H-4010 Debrecen, Hungary \\ ${ }^{5}$ Institute of Nuclear Medicine, Medical and Health Science Center, University of Debrecen, P.O. Box 12, H-4010 Debrecen, Hungary
}

(Received 21 January 2014; revised manuscript received 19 March 2014; published 13 May 2014)

\begin{abstract}
Strictly finite-range (SFR) potentials are exactly zero beyond their finite range. Single-particle energies and densities, as well as $S$-matrix pole trajectories, are studied in a few SFR potentials suited for the description of neutrons interacting with light and heavy nuclei. The SFR potentials considered are the standard cutoff Woods-Saxon (CWS) potentials and two potentials approaching zero smoothly: the SV potential introduced by Salamon and Vertse [Phys. Rev. C 77, 037302 (2008)] and the SS potential of Sahu and Sahu [Int. J. Mod. Phys. E 21, 1250067 (2012)]. The parameters of these latter potentials were set so that the potentials may be similar to the CWS shape. The range of the SV and SS potentials scales with the cube root of the mass number of the core like the nuclear radius itself. For light nuclei a single term of the SV potential (with a single parameter) is enough for a good description of the neutron-nucleus interaction. The trajectories are compared with a benchmark for which the starting points (belonging to potential depth zero) can be determined independently. Even the CWS potential is found to conform to this benchmark if the range is identified with the cutoff radius. For the CWS potentials some trajectories show irregular shapes, while for the SV and SS potentials all trajectories behave regularly.
\end{abstract}

DOI: 10.1103/PhysRevC.89.054609

PACS number(s): 21.10.Pc, 25.40.Dn, 87.57.uk

\section{INTRODUCTION}

We call the potentials that are exactly zero beyond a certain distance strictly finite-range (SFR) potentials. The conventional nuclear potentials are, in principle, not SFR potentials, but in practice, if the radial Schrödinger equation is solved numerically as is usual, a cutoff at a finite range is implied. Indeed, beyond this range $R_{\max }$ the numerical solution is to be matched at a finite distance $r=R_{\text {match }}\left(\geqslant R_{\max }\right)$ with the exact solution of the free-particle (or of the Coulomb) problem.

For instance, the most often quoted Woods-Saxon (WS) potential goes to zero in infinity, but, in numerical calculations, cutoff WS (CWS) potentials are used invariably. A disadvantage of the CWS potential is that the positions of the resonance poles do depend on the cutoff distance [1], which is an unphysical parameter of the calculation. To avoid this, a new form was introduced by Salamon and Vertse (SV) [1], which contains two terms, with one range parameter for each, and a relative strength of the two terms. The SV potential goes to zero smoothly. Its parameters can be adjusted so as to get a good fit to the WS shape except in the tail region, where they are necessarily different.

There is another motivation for using SFR potentials. It has been observed recently by Sahu and Sahu [2] that a faster approach of the nuclear potential to zero improves the barrier behavior of the interaction potential between heavy ions. They modified the form of the SV potential by introducing a diffuseness parameter $a_{s}$ to one of its terms. Here we refer to this potential as the SS potential. The SS potential was found to describe the elastic scattering and the fusion below the Coulomb barrier with the same parameters, while a WS form requires two different sets for these two processes [2].

However, the asymptotic density of the matter of nuclei is exponential, and the nucleon-nucleon interaction has a Yukawa tail. This physically substantiates the numerically untractable exponential falloff of the WS potential and casts some doubt on the use of the convenient tails of the SV and SS potentials. In this paper we examine the effect of the unphysical tail behavior of the SV potential and further study the trajectories of the $S$-matrix poles. The SV potential is a special case of the SS potential with $a_{s}=1$, and we extend the studies to $a_{s} \neq 1$. In fact, for very light nuclei the derivative term in the SV potential can be omitted, and the SS form becomes identical to an SV form, which has a single parameter, the range $\rho_{0}$.

In this work we consider nucleon potential problems. Because we disregard the Coulomb interaction, we can say that we deal with neutrons. We perform bound-state and resonance calculations, with an eye to scattering problems, but we need no absorptive terms. We study the cases of light nuclei with mass number $A_{T}<20$, as well as nuclei with much larger $A_{T}$ values. Light nuclei are important in fusion reactions taking place in the Sun. The nucleon optical potential of light nuclei is an ingredient of the description of the reactions producing the nuclides used in positron emission tomography (PET). ${ }^{1}$

\section{FUNCTIONAL FORMS OF THE POTENTIALS CONSIDERED}

The real term of the optical potential is almost exclusively of CWS form, and the spin-orbit part contains the derivative of a CWS form.

The CWS potential can be written as

$$
V^{\mathrm{CWS}}\left(r, R, a, R_{\max }\right)=-V_{0} f^{\mathrm{CWS}}\left(r, R, a, R_{\max }\right),
$$

\footnotetext{
${ }^{1}$ The standard reactions producing the most important positron emitters are ${ }^{14} \mathrm{~N}(p, \alpha){ }^{11} \mathrm{C},{ }^{13} \mathrm{C}(p, n){ }^{13} \mathrm{~N},{ }^{15} \mathrm{~N}(p, n){ }^{15} \mathrm{O}$, and ${ }^{18} \mathrm{O}(p, n){ }^{18} \mathrm{~F}$.
} 
with

$$
f^{\mathrm{CWS}}\left(r, R, a, R_{\max }\right)=\left(1+e^{\frac{r-R}{a}}\right)^{-1} \theta\left(R_{\max }-r\right),
$$

where the Heaviside step function $\theta(x)$ is unity for positive $x$ and zero otherwise. The CWS form factor $f^{\mathrm{CWS}}\left(r, R, a, R_{\max }\right)$ has two physical parameters, the radius $R$ and the diffuseness $a$. The third parameter, the cutoff radius $R_{\max }$, should have no physical significance, but, owing to the jump at the finite $R_{\max }$, its derivative does not exist there, and that has implications.

It was shown earlier [1] that the positions of broad resonances in a CWS potential do depend on the value of the cutoff radius $R_{\max }$. Certain sections of the pole trajectories (mainly the starting regions) have been found to be sensitive to the value of $R_{\max }[3,4]$. Thus, the cutoff radius $R_{\max }$ is an important, though nonphysical, parameter of the CWS form.

The SV potential [1] recommended by two of us instead of the CWS potential has the form [3]

$$
V^{\mathrm{SV}}(r)=-V_{0} f^{\mathrm{SV}}\left(r, c, \rho_{0}, \rho_{1}\right),
$$

in which $V_{0} \geqslant 0$ and $f^{\mathrm{SV}}\left(r, c, \rho_{0}, \rho_{1}\right)$ is a linear combination of the function

$$
f(r, \rho)=e^{\frac{r^{2}}{r^{2}-\rho^{2}}} \theta(\rho-r),
$$

and a term containing the derivative, with respect to $r$, of the first factor,

$$
f^{\prime}(r, \rho)=-\frac{2 r \rho^{2}}{\left(r^{2}-\rho^{2}\right)^{2}} e^{\frac{r^{2}}{r^{2}-\rho^{2}}} \theta(\rho-r) .
$$

Note that the function in Eq. (4) is a variant of the well-known functions of compact support, $C^{\infty}$, defined in the book by Bremmermann [5] and sometimes called bump functions. The radial factor thus contains three adjustable parameters,

$$
f^{\mathrm{SV}}\left(r, c, \rho_{0}, \rho_{1}\right)=f\left(r, \rho_{0}\right)-c f^{\prime}\left(r, \rho_{1}\right),
$$

in which $\rho_{0}$ and $\rho_{1}$ need not be the same, and, for the second term to be attractive, the coefficient $c$ is non-negative. The potential $V^{\mathrm{SV}}(r)$ goes to zero smoothly, and, if $\rho_{0}>\rho_{1}$, it vanishes at $\rho_{0}$; furthermore, for $r \geqslant \rho_{0}$, it is zero, together with all its derivatives. Thus, the SV potential has the attractive mathematical property that its derivative exists in the whole $r \in(0, \infty)$ region. A drawback is, however, that it is not analytic because at $\rho_{0}$ the Taylor series is not equal to the function. Nevertheless, it has turned out to be useful in quantum electrodynamics, too, as a compactly supported smooth regulator function [6].

The formula of the SS potential [2] is analogous to Eq. (6),

$$
f^{\mathrm{SS}}\left(r, c, \rho_{0}, \rho_{1}, a_{s}\right)=f\left(r, \rho_{0}\right)-c f^{\prime}\left(r, \rho_{1}, a_{s}\right),
$$

where

$$
f^{\prime}\left(r, \rho_{1}, a_{s}\right)=-\frac{2 r \rho_{1}^{2}}{\left(r^{2}-\rho_{1}^{2}\right)^{2}} e^{\frac{a_{s} r^{2}}{r^{2}-\rho_{1}^{2}}} \theta\left(\rho_{1}-r\right),
$$

with $a_{s}$ being the extra diffuseness parameter. When $a_{s}=1$, the SS form coincides with the SV potential (3). By using $a_{s} \neq 1$, one naturally has more freedom in choosing the shape of the potential. With the usual choice $\rho_{0}>\rho_{1}$, the range of the SS potential is also $\rho_{0}$. The SS form has the same attractive mathematical features as the SV potential.
Let us return for a while to the original SV form. If we want the shape of the SV form to be similar to the WS shape as much as possible, we should fit its parameters to the CWS shape $f^{\mathrm{CWS}}$. To this end, we can minimize

$$
\int_{0}^{\rho_{0}}\left[f^{\mathrm{SV}}\left(r, c, \rho_{0}, \rho_{1}\right)-f^{\mathrm{CWS}}\left(r, R, a, R_{\max }\right)\right]^{2} d r .
$$

The integration in Eq. (9) can be performed by a quadrature of $m$ equidistant mesh points $r_{i}=i h$ over the range of the integration, so that what is minimized is

$$
\Delta\left(\rho_{0}, \rho_{1}, c\right)=\sum_{i=1}^{m}\left[f^{\mathrm{SV}}\left(r_{i}, c, \rho_{0}, \rho_{1}\right)-f^{\mathrm{CWS}}\left(r_{i}, R, a, R_{\max }\right)\right]^{2} .
$$

\section{GLOBAL PARAMETER SETS FOR OPTICAL POTENTIALS}

In this section we construct SV potentials that approximate the real parts of some well-known global nucleon optical model potentials and test their performance. The real parts of all global potentials are of CWS shape. Their geometrical shapes are generally fixed, and their energy dependence is restricted to the strength parameters. The spin-orbit part for a particle with spin $s=\frac{1}{2} \hbar$ is

$$
V_{\mathrm{so}}^{\mathrm{CWS}}\left(r, R_{\mathrm{so}}, a_{\mathrm{so}}, R_{\mathrm{max}}\right)=V_{\mathrm{so}}^{\mathrm{CWS}} h_{\mathrm{CWS}}\left(r, R_{\mathrm{so}}, a_{\mathrm{so}}, R_{\max }\right) 2(\mathbf{l} \cdot \mathbf{s}) \text {, }
$$

with a radial form

$$
h_{\mathrm{CWS}}\left(r, R, a, R_{\max }\right)=-\frac{1}{r} f_{\mathrm{CWS}}^{\prime}\left(r, R, a, R_{\max }\right),
$$

in which the derivative of the central potential,

$$
f_{\mathrm{CWS}}^{\prime}\left(r, R, a, R_{\max }\right)=-\frac{e^{\frac{r-R}{a}}}{a\left[1+e^{\frac{r-R}{a}}\right]^{2}} \theta\left(R_{\max }-r\right),
$$

appears.

The spin-orbit term of the SV potential may be defined analogously,

$$
V_{\mathrm{so}}^{\mathrm{SV}}\left(r, c, \rho_{0}, \rho_{1}\right)=V_{\mathrm{so}}^{\mathrm{SV}} h_{\mathrm{SV}}\left(r, c, \rho_{0}, \rho_{1}\right) 2(\mathbf{l} \cdot \mathbf{s}),
$$

with

$$
h_{\mathrm{SV}}\left(r, c, \rho_{0}, \rho_{1}\right)=-\frac{1}{r} f_{\mathrm{SV}}^{\prime}\left(r, c, \rho_{0}, \rho_{1}\right)
$$

The mass-number dependence of the global potentials is borne generally by the radii such that $R_{\alpha}=r_{\alpha, 0} A_{T}^{1 / 3}$, where $\alpha$ labels any of the potential terms.

Classical nucleon potential sets were given by Perey [7] and by Becchetti and Greenlees [8] a long time ago, and they are relied on in recent studies [9] as well. A recent attempt for the derivation of a new $\alpha$-nucleus potential was made by Mohr and co-workers [10]. In this work, however, we restrict ourselves to the Perey and Becchetti-Greenlees parameters for simplicity.

To construct global SV potentials, we search for the minimum of the squared deviations in Eq. (9) as a function of the mass number $A_{T}$ and calculate the best-fit SV parameters 


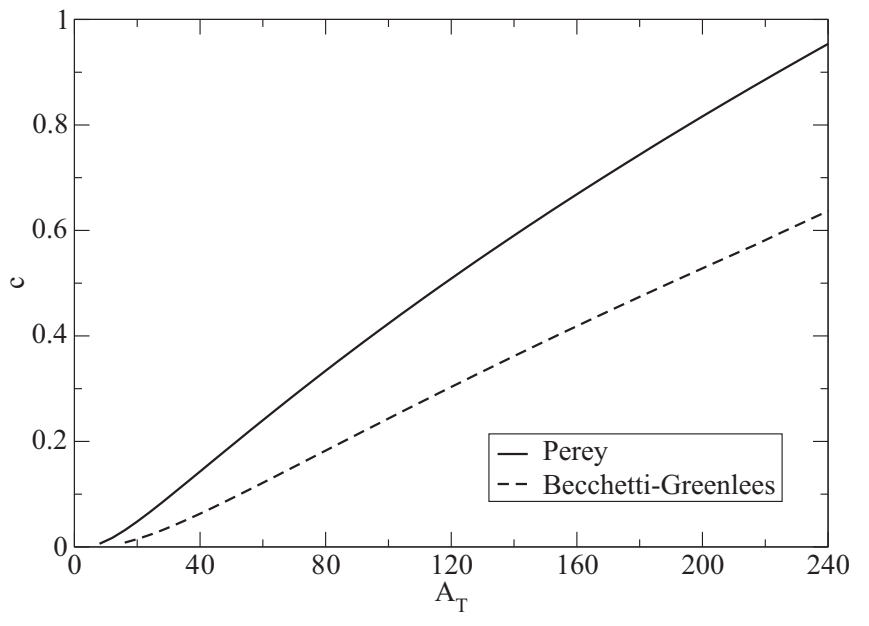

FIG. 1. Dependence of the mixing coefficient $c$ on the target mass number $A_{T}$ for two global parameter sets.

as a function of $A_{T}$. For medium-heavy and heavy nuclei, the SV potential reproduces the CWS shape quite well, and its $A_{T}$ dependence is regular. The mixing coefficient $c$ decreases with decreasing $A_{T}$, as seen in Fig. 1. In the region of light nuclei, however, the best-fit SV form has a strange, irregular shape. We can avoid this by requiring that the derivative of the SV form be similar to the derivative of the WS shape:

$$
\begin{aligned}
\Delta\left(\rho_{0}, \rho_{1}, c\right)= & \sum_{i=1}^{m}\left[f^{\mathrm{SV}}\left(r_{i}, c, \rho_{0}, \rho_{1}\right)-f^{\mathrm{CWS}}\left(r_{i}\right)\right]^{2} \\
& +\lambda\left[f^{\mathrm{SV}^{\prime}}\left(r_{i}, c, \rho_{0}, \rho_{1}\right)-f^{\mathrm{CWS}^{\prime}}\left(r_{i}\right)\right]^{2} .
\end{aligned}
$$

The Lagrange multiplier $\lambda$ was determined empirically. (Here we suppressed the parameters of the CWS potential, which were kept fixed.) With a value of $\lambda=25 \mathrm{fm}^{2}$, the fitted SV potential became reasonably smooth and similar to the CWS shape we want to approximate.

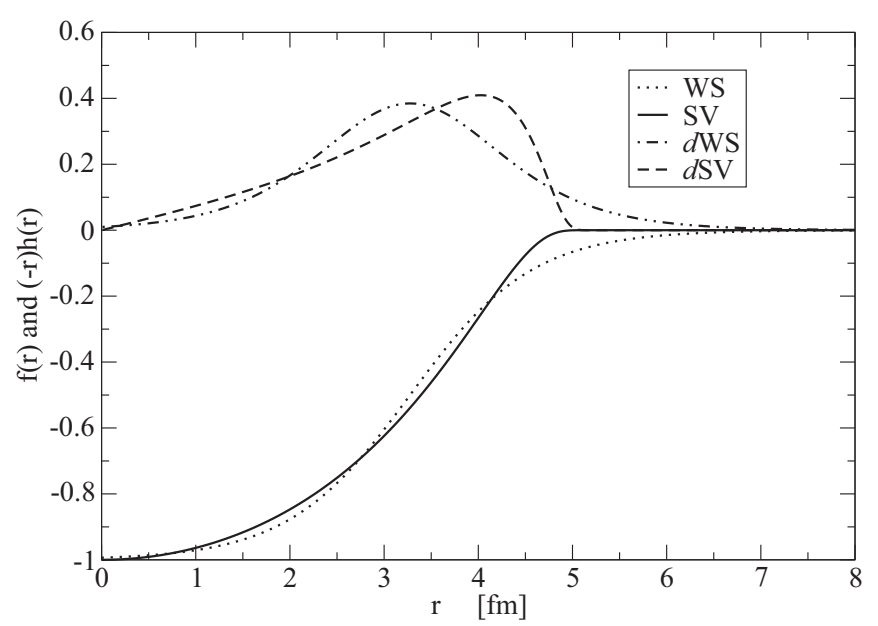

FIG. 2. Radial shapes of Perey's WS and the SV $(c=0)$ potentials and their derivatives for ${ }^{18} \mathrm{~F}+n$. Derivatives appear in the spin-orbit terms in Eqs. (12) and (15).
TABLE I. Geometrical parameters of the WS and the SV potentials for ${ }^{13} \mathrm{~N},{ }^{15} \mathrm{O}$, and ${ }^{18} \mathrm{~F}$. All distances are in units of $\mathrm{fm}$.

\begin{tabular}{lccccc}
\hline \hline$A_{T}$ & $r_{0}=R / A_{T}^{1 / 3}$ & $R$ & $a$ & $\rho_{0} / A_{T}^{1 / 3}$ & $\rho_{0}$ \\
\hline${ }^{13} \mathrm{~N}$ & 1.25 & 2.94 & 0.65 & 2.037 & 4.79 \\
${ }^{15} \mathrm{O}$ & 1.25 & 3.08 & 0.65 & 2.031 & 5.01 \\
${ }^{18} \mathrm{~F}$ & 1.25 & 3.28 & 0.65 & 2.022 & 5.30 \\
\hline \hline
\end{tabular}

The range $\rho_{0}$ of the $S V$ potential scales with $A_{T}^{1 / 3}$, while the difference $\rho_{0}-\rho_{1}$ is proportional to the diffuseness $a$ of the CWS potential. The parameters of the Perey potential [7] are $r_{0}=1.25 \mathrm{fm}$ and $a=0.65 \mathrm{fm}$, and the best-fit SV parameters are $\rho_{0}=1.85 A_{T}^{1 / 3} \mathrm{fm}, \rho_{0}-\rho_{1}=3.2 a$, and $c=-0.051+$ $0.0051 A_{T}-3.9 \times 10^{-6} A_{T}^{2}$; thus, for small $A_{T}, c$ becomes very small. For the Becchetti-Greenlees [8] geometry $\left(r_{0}=\right.$ $1.17 \mathrm{fm}$ and $a=0.75 \mathrm{fm}$ ), the best-fit SV parameters relate to the CWS parameters very similarly; namely, their values are $\rho_{0}=1.86 A_{T}^{1 / 3} \mathrm{fm}, \rho_{0}-\rho_{1}=2.8 a$, and $c=-0.055+$ $0.003 A_{T}-7.0 \times 10^{-7} A_{T}^{2}$.

As a light system, let us consider ${ }^{18} \mathrm{~F}+n$. For the Perey geometry, the best-fit SV parameters are $\rho_{0}=5.084 \mathrm{fm}, \rho_{1}=$ $3.244 \mathrm{fm}$, and $c=0.040$, while for the Becchetti-Greenlees geometry, we get $\rho_{0}=4.957 \mathrm{fm}, \rho_{1}=2.728 \mathrm{fm}$, and $c=$ 0.011 . This again shows that for light nuclei $c$ is practically zero, and it is reasonable to take $c=0$.

In Fig. 2 we compare the shape of Perey's WS potential and its derivative with the SV potential (with $c=0$ ) and its derivative for the ${ }^{18} \mathrm{~F}+n$ system. The WS parameters are listed in Table I. The ratio $\rho_{0} / A_{T}^{1 / 3}$ is almost constant with a value of $\sim 1.6 r_{0}$. One can see that the radial shape of the WS potential is approximated reasonably well by the first term of the SV form with a single adjustable parameter, $\rho_{0}$. Now $\rho_{0}$ must play the role of both the radius and the diffuseness of the WS potential. Of course, the SV curves deviate most from the WS curves at large distances.

\section{SINGLE-PARTICLE ENERGIES FOR LIGHT NUCLEI}

It is interesting to see how the differences between the potentials influence the single-particle energies. In Table II we show the neutron single-particle energies $\epsilon_{n l j}$ calculated for the core nucleus ${ }^{18} \mathrm{~F}$, with Perey's WS geometry $\left(V_{0}^{\mathrm{CWS}}=\right.$ $60 \mathrm{MeV}, r_{0}=1.25 \mathrm{fm}, a=0.65 \mathrm{fm}, R_{\max }=15 \mathrm{fm}$, and $V_{\mathrm{so}}^{\mathrm{CWS}}=28 \mathrm{MeV}$ ). For the fitted SV potential we used two

TABLE II. ${ }^{18} \mathrm{~F}+n$ single-particle energies (in $\mathrm{MeV}$ ) in the CWS potential and in the fitted SV potential with one central term.

\begin{tabular}{lccc}
\hline \hline$i=\{n, l, j\}$ & \multirow{2}{*}{$\epsilon_{i}(\mathrm{CWS})$} & \multicolumn{2}{c}{$\epsilon_{i}(\mathrm{SV})$} \\
\cline { 3 - 4 } & & $V_{\mathrm{so}}^{\mathrm{SV}}=28 \mathrm{MeV}$ & $V_{\mathrm{so}}^{\mathrm{SV}}=30 \mathrm{MeV}$ \\
\hline $0 s_{1 / 2}$ & -38.926 & -38.119 & -38.119 \\
$0 p_{3 / 2}$ & -23.998 & -23.568 & -23.611 \\
$0 p_{1 / 2}$ & -22.067 & -21.729 & -21.640 \\
$0 d_{5 / 2}$ & -8.985 & -8.962 & -9.049 \\
$1 s_{1 / 2}$ & -7.697 & -7.699 & -7.699 \\
$0 d_{3 / 2}$ & -5.779 & -5.901 & -5.770 \\
\hline \hline
\end{tabular}


TABLE III. ${ }^{13} \mathrm{~N}+n$ single-particle energies (in $\mathrm{MeV}$ ) in the CWS potential and in the corresponding $\mathrm{SV}$ potential with one central term and $V_{\text {so }}^{\text {SV }}=30 \mathrm{MeV}$.

\begin{tabular}{lrr}
\hline \hline$i=\{n, l, j\}$ & $\epsilon_{i}(\mathrm{CWS})$ & $\epsilon_{i}(\mathrm{SV})$ \\
\hline $0 s_{1 / 2}$ & -35.045 & -36.746 \\
$0 p_{3 / 2}$ & -18.620 & -20.368 \\
$0 p_{1 / 2}$ & -16.318 & -17.958 \\
$0 d_{5 / 2}$ & -3.067 & -4.247 \\
$1 s_{1 / 2}$ & -3.400 & -3.400 \\
$0 d_{3 / 2}$ & -0.003 & -0.548 \\
\hline \hline
\end{tabular}

values for the spin-orbit strength. In the first case the spin-orbit term (14) was used with $V_{\text {so }}^{\mathrm{SV}}=V_{\text {so }}^{\mathrm{CWS}}=28 \mathrm{MeV}$. However, as seen in Fig. 2, the shape of the derivative differs somewhat from that of the standard form. Therefore, to achieve similar spinorbit splitting, in the second case we used a bit stronger $\left(V_{\mathrm{so}}^{\mathrm{SV}}=\right.$ $30 \mathrm{MeV}$ ) value for the spin-orbit strength.

One can see that, with the larger spin-orbit strength, the SV energies are pretty close to the CWS energies. The differences are largest for the deepest orbits. Similar behaviors were found for the other two residual nuclei. In Table III we present the calculated single-particle energies for ${ }^{13} \mathrm{~N}+n$, in which the $d_{3 / 2}$ orbit is very close to the threshold.

We can conclude that for light nuclei the one-term SV potential is a good phenomenological form, which reproduces the spectra obtained with the conventional WS potentials, although the shape of its derivative is somewhat different from that of the CWS potential.

The wave functions produced by the two potentials are most conveniently compared through the neutron densities,

$$
\rho(r)=\sum_{i} v_{i}^{2}\left[\frac{u_{i}(r)}{r}\right]^{2},
$$

where $i=\left\{n_{i}, l_{i}, j_{i}\right\}$ runs over the occupied orbits, $u_{i}(r)$ denotes the single-particle radial wave functions, and $v_{i}^{2}$ is the occupation number. It is assumed that the lowest-lying orbits

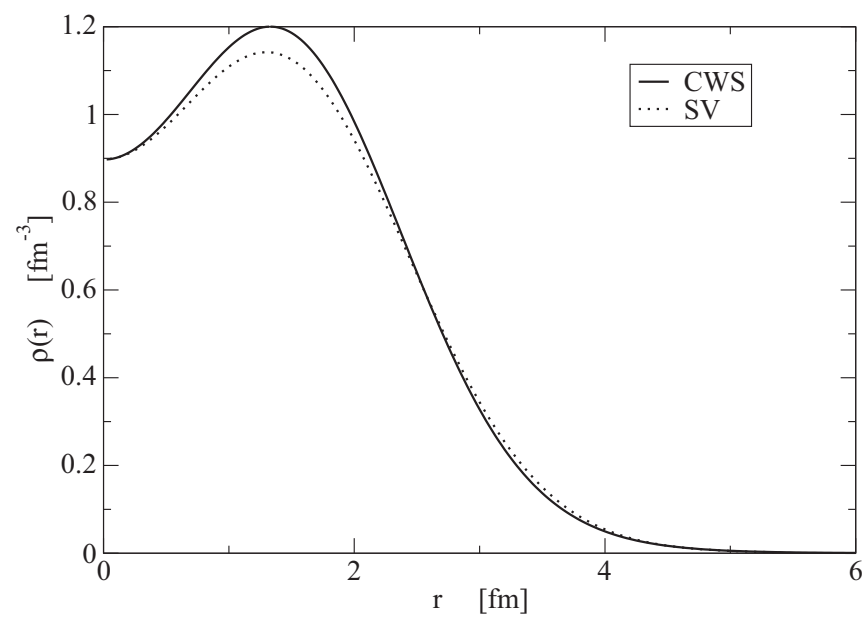

FIG. 3. Radial shapes of the neutron densities for the nucleus ${ }^{18} \mathrm{~F}$ in CWS and in SV potentials. are fully occupied, i.e., $v_{i}^{2}=2 j_{i}+1$. In Fig. 3 we compare the neutron densities calculated for the nucleus ${ }^{18} \mathrm{~F}$ in CWS and in SV potentials. The difference between the two densities is largest at the peak of the densities produced by the two deeply bound orbits, where the energies are deeper in the CWS potential. In the surface, where the CWS and SV potentials do differ appreciably, the two densities do not differ significantly. For $r>4 \mathrm{fm}$, the two curves can hardly be distinguished because the tail of the density is mostly determined by the single-particle energies being close to the Fermi level, which are very similar in the two potentials.

\section{THE CWS POTENTIAL IMITATED BY THE SS FORM}

The SS modification only matters for heavier systems, and we consider ${ }^{208} \mathrm{~Pb}+n$. First we show the effect of $a_{s} \neq 1$ on a potential whose SV parameters $\rho_{0}, \rho_{1}$, and $c$ were adjusted to the CWS shape [3,4]. In Fig. 4 we can see that $a_{s}>1$ smooths the SV potential in the region around $\rho_{1}$, where the SV curve shows a bend, while $a_{s}<1$ sharpens the bend, and even an extra minimum shows up. Such an extra minimum (a pocket) was needed for the description of $\alpha$ decay from $\mathrm{Ra}$ isotopes in Ref. [11].

To determine the SS form that approximates the CWS potential best, we should fit all the four parameters of the SS potential simultaneously. We minimized the function

$$
\begin{aligned}
\Delta\left(\rho_{0}, \rho_{1}, a_{s}, c\right)= & \sum_{i=1}^{m}\left[f^{\mathrm{SS}}\left(r_{i}, c, \rho_{0}, \rho_{1}, a_{s}\right)-f^{\mathrm{CWS}}\left(r_{i}\right)\right]^{2} \\
& +\lambda\left[f^{\mathrm{SS}^{\prime}}\left(r_{i}, c, \rho_{0}, \rho_{1}, a_{s}\right)-f^{\mathrm{CWS}^{\prime}}\left(r_{i}\right)\right]^{2},
\end{aligned}
$$

with $\lambda=25 \mathrm{fm}^{2}$. The two potentials are shown in Fig. 5, and the parameters are given in the caption. The agreement is remarkable in spite of the SS potential having a minimum.

\section{POLE TRAJECTORIES IN SFR POTENTIALS}

Having indicated some practical aspects of using SFR potentials in nuclear problems, we now discuss the problem

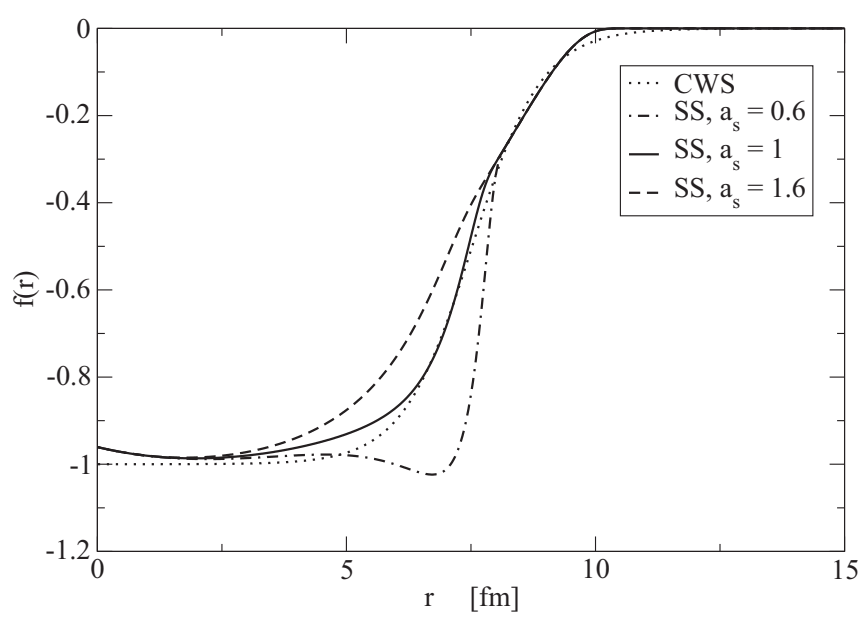

FIG. 4. Radial shapes of the CWS and SS potentials with different $a_{s}$ values for ${ }^{208} \mathrm{~Pb}+n$. 


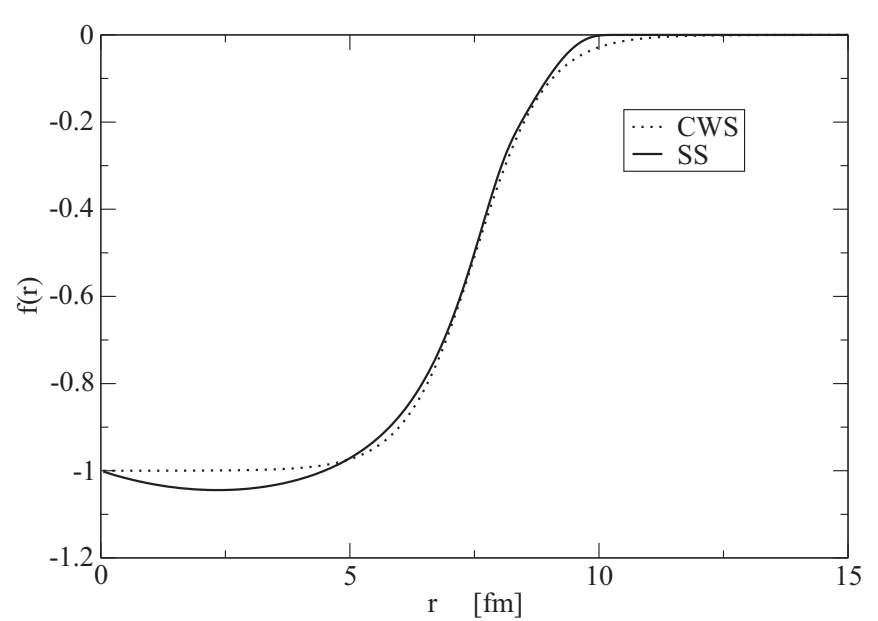

FIG. 5. Best-fit SS shape to the CWS shape for ${ }^{208} \mathrm{~Pb}+n$. WS parameters: $r_{0}=1.27 \mathrm{fm}, a=0.7 \mathrm{fm}$. SS parameters: $\rho_{0}=10.75$ $\mathrm{fm}, \rho_{1}=8.94 \mathrm{fm}, c=1.528, a_{s}=1.4$.

of pole trajectories. We remind the reader that the pole trajectories, especially in the region of broad resonances, are what makes the use of truncated potentials dangerous. Pole trajectories can be labeled conveniently by $n$, the number of nodes of the wave function defined where the pole belongs to a bound (or antibound) state. However, the trajectories can be found more easily at the other extreme, where the potential strength is nearly zero (at the "starting point"). Here the states are resonances with complex radial wave functions, whose real, as well as imaginary, parts have infinite numbers of zeros. Orbits with low $n$ values are important in nuclear structure calculations and in low-energy nucleon scattering. In heavy-ion reactions larger $n$ values occur. In the present work we restrict ourselves to the $s$-wave case. Analytical results are available for the square-well potential in the work of Nussenzveig [12] as was discussed by some of us recently [4]. However, because we are concerned with less special potentials, which cannot be treated analytically, we reconsider approximate analytical formulas for the starting points of the trajectories given in the literature. We are interested in where these are valid and how they can be treated numerically.

\section{A. Formulas for the starting points}

The $l=0$ states in the SFR potential

$$
V(r)=V_{0} \theta(\mathcal{R}-r)\left[(\mathcal{R}-r)^{\sigma}+\cdots\right]
$$

are discussed by Newton in his book [13] [see Eq. (12.98) on p. 361 there]. Here $\sigma>0, \theta(x)$ denotes the Heaviside step function, and the square bracket contains a truncated expansion in terms of $\mathcal{R}-r$. In Eq. (12.102) on p. 362, Newton gives the real and imaginary parts of the starting point $k_{n}=k_{n}^{R}-\mathrm{i} k_{n}^{I}$ of the trajectory of the $n$th pole of the $S$ matrix as

$$
k_{n}^{R}=\frac{n \pi}{\mathcal{R}}+O(1)
$$

and

$$
k_{n}^{I}=\frac{\sigma+2}{2 \mathcal{R}} \ln (n)+O(1) .
$$

The starting point of the pole trajectory is in the fourth quadrant of the $k$ plane, and, by definition, it belongs to $V_{0}=0$. Equations (20) and (21) are especially useful for large $n$ values, where the $\mathrm{O}(1)$ terms in the equations can be neglected, but it is interesting to see how they are fulfilled for lower $n$. Equation (20) depends linearly on $n$ with a slope

$$
A_{1}=\frac{\pi}{\mathcal{R}}
$$

Regge pointed out [14] that a relation similar to Eq. (20) is valid for the moduli of the starting wave number values:

$$
\left|k_{n}\right|=\frac{n \pi}{\mathcal{R}}+\mathrm{O}(1)=A_{1} n+\mathrm{O}(1)
$$

\section{B. Test with Newton's potential}

For a potential of the form of Eq. (19), the asymptotic expressions (20), (21), and (23) offer convenient tests of our numerical procedure for very large $n$ values. Inaccuracies may come from approximating $V_{0}=0$ by a small finite value, from truncation errors in the numerical integration of the differential equation, and from rounding errors throughout the numerical calculations. We reduced the rounding errors by using extended precision floating-point arithmetics. We used Ixaru's method [15] for the numerical integration of the radial equation, and we calculated the position of the pole of the $S$ matrix using the computer code ANTI [16].

We chose a potential of the form of Eq. (19) with $\sigma=1$,

$$
V(r)=-V_{0} \theta(\mathcal{R}-r)(\mathcal{R}-r),
$$

which is attractive if $V_{0}>0$, and chose $V_{0}=0.005 \mathrm{MeV}$ and $\mathcal{R}=10 \mathrm{fm}$.

We calculated the starting values $k_{n}$ for the $n=1, \ldots, 98$ trajectories and fitted the $k_{n}^{R}$ values by a first-order polynomial of $n$, i.e.,

$$
y(n)=a_{0}+a_{1} n .
$$

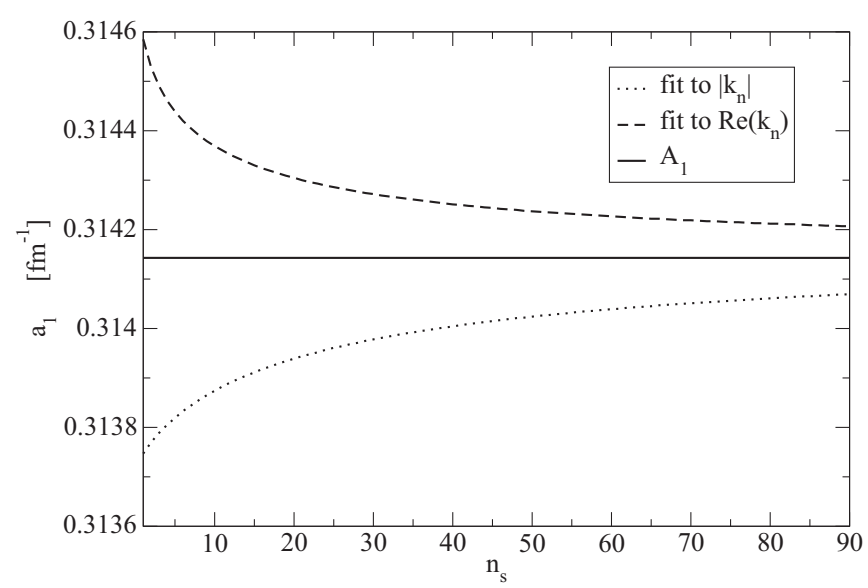

FIG. 6. Dependence of the slope of the fitted line on the lower cut value of the node number $n_{s}$ for a potential in Eq. (24) with a range of $\mathcal{R}=10 \mathrm{fm}$. 


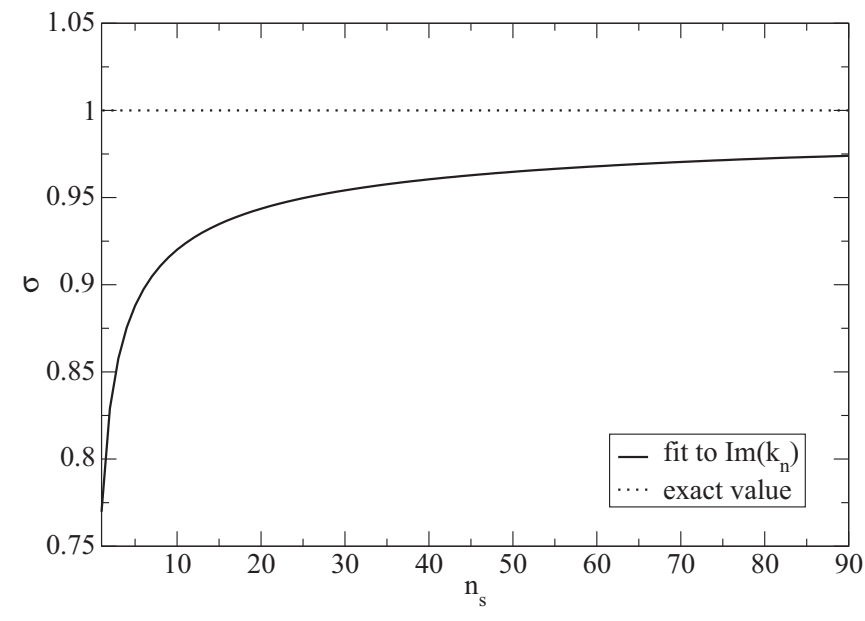

FIG. 7. Convergence of the fitted $\sigma$ to the exact value (dotted line) obtained by using the lower cut value of the node number $n_{s}$ for a potential of Eq. (24).

Because in Eq. (20) we have an unknown O(1) term (the actual value of this is reflected by $a_{0}$ ), we applied a lower cut value $n_{s}$ in our data and performed the fitting for a number of $n \in$ $\left\{n_{s}, n_{s}+1, \ldots, n_{u}\right\}$, with $n_{u}=98$ fixed and $n_{s}$ varied. We can thus estimate the value of $a_{1}$ for each $n_{s}$ and compare it with $A_{1}=\pi / \mathcal{R}=0.31416 \mathrm{fm}^{-1}$ obtained from Eq. (22). In Fig. 6 the ordinate shows the deduced slope, with the horizontal line $A_{1}=\pi / 10 \mathrm{fm}^{-1}$, to which the fitted values of $a_{1}$ should converge for large $n_{s}$. The dashed line connects the $a_{1}$ values resulting from the fit to $k_{n_{s}}^{R}$. It is seen that the estimate for the range has three accurate digits even for $n_{s}=1$.

To check the validity of Eq. (23), we fitted a linear function to the moduli of the starting wave number values calculated, and followed a procedure similar to that for $k_{n}^{R}$. The dotted line in Fig. 6 shows the slopes obtained as a function of $n_{s}$. Now the fitted slope $a_{1}$ approaches the horizontal line from below and yields an estimate of similar accuracy. The results of these tests show that the small final value of $V_{0}$ we use provides a reasonable estimate for the starting value of the pole trajectory.

To check Eq. (21) for the imaginary part of $k_{n}$, we introduce the variable $x=\ln (n)$ and fit $k_{n}^{I}=a_{1} x+a_{0}$ for the same sets of $n=n_{s}, \ldots, 98$ points, with $n_{s}=1, \ldots, 97$. From the slope $a_{1}$ obtained, we can calculate $\sigma=2 a_{1} \mathcal{R}-2$ as a function of $n_{s}$ using the actual value of $\mathcal{R}$. Figure 7 shows that this $\sigma$ converges to 1 as it should, but rather slowly.

\section{Cutoff Woods-Saxon form}

The trajectories of the $S$-matrix poles were calculated for two SFR potentials for a heavy nucleus ${ }^{208} \mathrm{~Pb}$ in Refs. [3,4]. Certain features found in Ref. [4] indicate that the relationship (20) might hold for the CWS and even for the SV potentials.

The asymptotic behavior of the CWS potential for $r<R_{\max }$ may be approximated by a Taylor series around $r=\mathcal{R}=R_{\max }$ cut after the first term,

$$
-V_{0} f^{\mathrm{CWS}}\left(r, R, a, R_{\max }\right) \approx D+\left(R_{\max }-r\right) \frac{D}{a},
$$

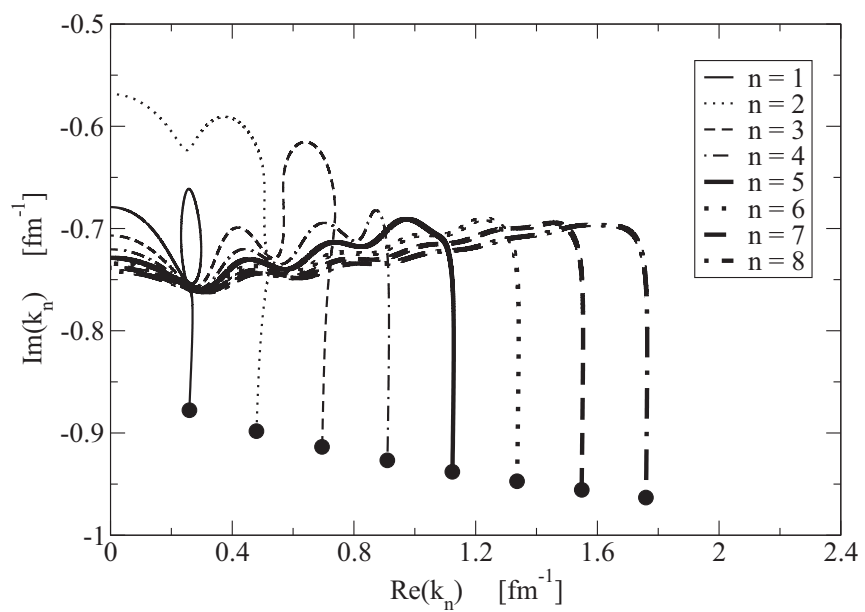

FIG. 8. Pole trajectories for a CWS potential with $R_{\max }=15 \mathrm{fm}$ for $l=0$ and $n=1, \ldots, 8$ for ${ }^{18} \mathrm{~F}$. The solid circles denote the starting points of the trajectories with $V_{0}=0.005 \mathrm{MeV}$.

where $D=-V_{0} e^{\left(R-R_{\max }\right) / a}$. The second term corresponds to a $\sigma=1$ version of Newton's potential studied before, but now we have an additional first term, which does not depend on $r$. Thus, not even an approximation to the CWS potential has exactly the form of Eq. (19). However, with the usual choice of $R_{\max } \geqslant R+6 a$, the value of the constant $|D| \leqslant 0.0025 \times V_{0}$; thus, the first term is not very large.

Because for a heavy nucleus, a crucial difference has been observed between the pole trajectories of the continuous SV potential and the discontinuous CWS potential [3,4], here we extend these calculations to light nuclei and to the SS potential.

For ${ }^{208} \mathrm{~Pb}$, it has been found [4] that the starting points of the $l=0$ resonant trajectories follow Newton's rule in Eq. (20) approximately if the $n$ value is not very small even though the asymptotic behavior of the potential (26) differs slightly from Eq. (19). Figure 8 shows the trajectories of a few poles of the ${ }^{18} \mathrm{~F}+n$ system in the CWS well with

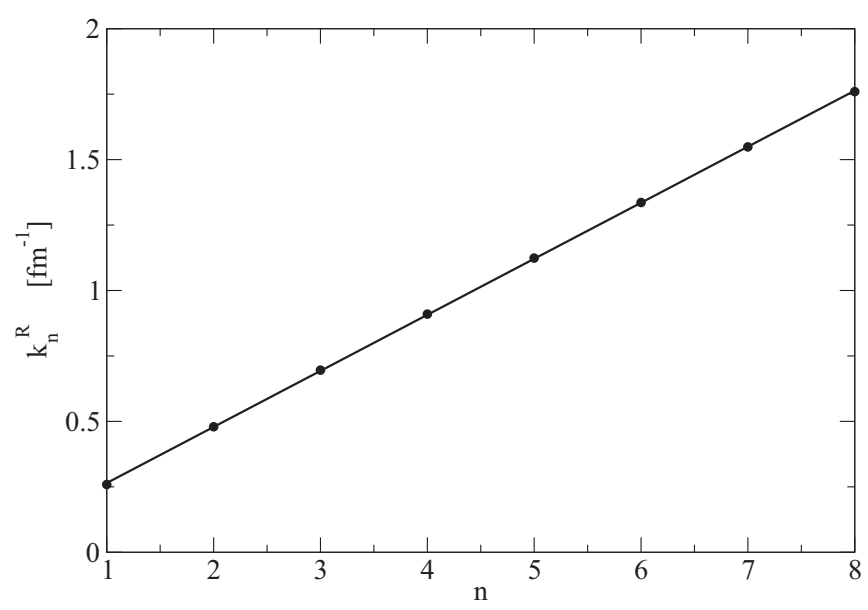

FIG. 9. The line is the linear function fitted to the $k_{n}^{R}$ values (dots) of the pole trajectories with node numbers $n=1, \ldots, 8$ for a CWS potential for ${ }^{18} \mathrm{~F}$. These values correspond to the abscissas of the solid circles in Fig. 8. The fit results in a range $\mathcal{R}=14.67 \mathrm{fm}$. 


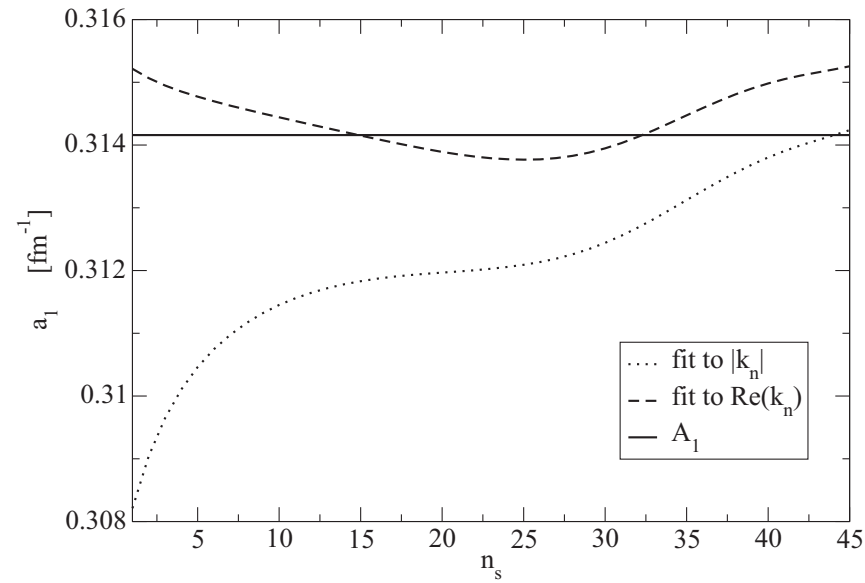

FIG. 10. Dependence of the slope of the fitted line on the lower cut value of the node number $n_{s}\left(n_{s}=1, \ldots, n_{u}-1\right.$ and $\left.n_{u}=48\right)$ for a CWS potential with $R_{\max }=10 \mathrm{fm}$.

parameters $r_{0}=1.25 \mathrm{fm}, a=0.65 \mathrm{fm}$, and $R_{\max }=15 \mathrm{fm}$. The results are similar to those for ${ }^{208} \mathrm{~Pb}$ even in that there is a loop in the $n=1$ trajectory but nowhere else. Figure 9 shows the straight line fitted to $k_{n}^{R}$ for node numbers $n=1, \ldots, 8$. From its slope Eq. (22) predicts $\mathcal{R}=14.67 \mathrm{fm}$, which agrees reasonably well with the cutoff radius used, $R_{\max }=15 \mathrm{fm}$ $\left(|D|=1.4 \times 10^{-8} V_{0}\right)$.

We studied the behavior of the trajectories further by setting the cutoff radius shorter, $R_{\max }=10 \mathrm{fm}\left(|D|=3.2 \times 10^{-5} V_{0}\right)$. In Fig. 10 we examine the validity of Eqs. (20) and (23) for the CWS potential by a test similar to that shown in Fig. 6 . Now the two curves do not converge smoothly into a constant. The agreement of the slope $a_{1}$ with the exact value is reduced to two decimal digits, and, as a function of $n$, it oscillates around $\pi / R_{\max }$. Thus, we can still state that Eqs. (20) and (23) are approximately satisfied by a CWS potential as well. The relationship for the imaginary part, Eq. (21), however, is not satisfied at all. There is no region where the deduced $\sigma$ would be more or less constant. It looks that Eq. (26) is too approximate to cause Eq. (21) to be fulfilled.

\section{Pole trajectories in SV and in SS potentials}

The pole trajectories for the SV potential behave absolutely regularly, with no loops and ripples (Fig. 11), in contrast to the CWS potential. The starting values $k_{n}^{R}$ can be fitted very well by a straight line as seen in Fig. 12. From its slope and Eq. (20) one can derive $\mathcal{R}=5.17 \mathrm{fm}$, which is just a bit less than the value of the range parameter $\rho_{0}=5.3 \mathrm{fm}$. Similar behavior was found before for ${ }^{208} \mathrm{~Pb}$ in Ref. [4]. We conclude that the relation in Eq. (20) is fulfilled approximately for SV and SS potentials in spite of their asymptotic behavior being different from Eq. (19). Thus, Eq. (20) is still useful for estimating the pole positions. Remember that for the SV and SS potentials the Taylor expansion at $\rho_{0}$ is not equal to the function, because all derivatives are zero at that point.

For two-term SV potentials $(c \neq 0)$, the starting values of the pole trajectories were studied in Ref. [3] for ${ }^{16} \mathrm{O}$ and

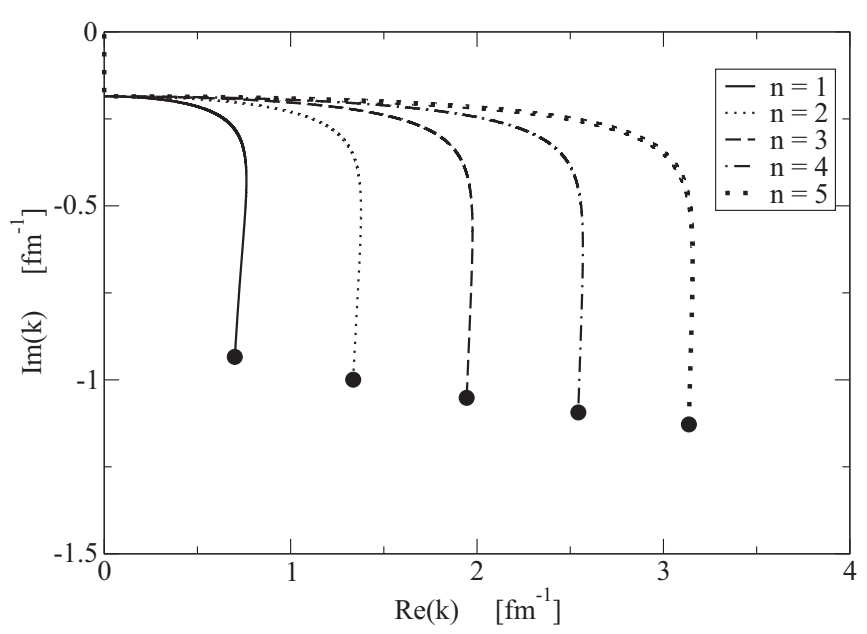

FIG. 11. Pole trajectories for a SV potential with $\rho_{0}=5.3 \mathrm{fm}$ for $l=0$ and $n=1, \ldots, 5$ for ${ }^{18} \mathrm{~F}$. The solid circles denote the $k_{n}$ points calculated with $V_{0}=0.005 \mathrm{MeV}$.

for ${ }^{208} \mathrm{~Pb}^{2}$ Now these studies may be extended to the SS potentials of various $a_{s}$. If the SS potential obeyed Newton's relation (19), the starting regions should be independent of $a_{s}$ and should coincide with the SV trajectory. Because, however, Eq. (19) does not hold even for the SV potential, we expect a dependence.

We consider a heavy core, where the derivative term is important: the case of ${ }^{208} \mathrm{~Pb}+n$. We choose $l=0$, analyze the SV potential that approximates the CWS potential of parameters $R=7.525 \mathrm{fm}$ and $a=0.7 \mathrm{fm}\left(\rho_{0}=10.963 \mathrm{fm}\right.$, $\rho_{1}=8.328 \mathrm{fm}$, and $\left.c=0.997\right)$, and repeat the calculation for

${ }^{2}$ In Ref. [3] it was conjectured that, for low node numbers, the $k_{n}^{R}-k_{n-1}^{R}$ is determined by $\frac{1}{2}\left(\rho_{0}+\rho_{1}\right)$. Later it turned out that this result was just an accident. The starting point depends only on $\rho_{0}$, where the potential vanishes.

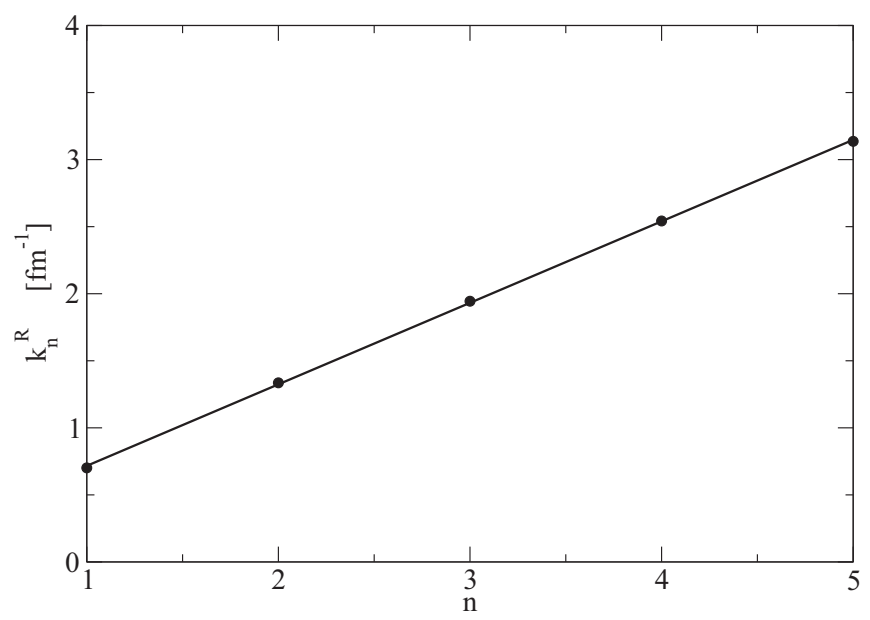

FIG. 12. Fit to the $k_{n}^{R}$ values of the solid circles in Fig. 11, with node numbers $n=1, \ldots, 5$ for a single-term SV potential for ${ }^{18} \mathrm{~F}$. The range deduced from the slope $a_{1}$ is $\mathcal{R}=5.17 \mathrm{fm}$. 


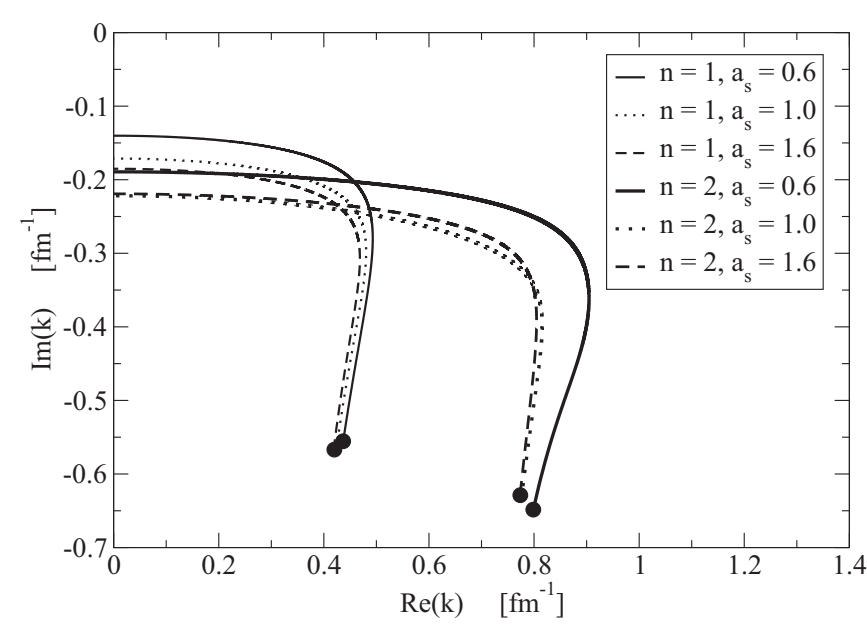

FIG. 13. Pole trajectories in SS potentials with different $a_{s}$ values. The value $a_{s}=1.0$ corresponds to the SV potential. The solid circles denote the $k_{n}$ values calculated with $V_{0}=0.005 \mathrm{MeV}$.

SS potentials of $a_{s}=0.6$ and 1.6 (Fig. 13). One can see that the three curves belonging to the same $n$ do not coincide, and nor do their starting points, but they slightly depend on $a_{s}$. This weak dependence may be attributed to departures from Eqs. (20) and (21) for low $n$.

We calculated the starting $k_{n}$ values for the best-fit SS shape to the same CWS shape for ${ }^{208} \mathrm{~Pb}+n$ (WS parameters, $r_{0}=$ $1.27 \mathrm{fm}, a=0.7 \mathrm{fm}$; SS parameters, $\rho_{0}=10.75 \mathrm{fm}, \rho_{1}=$ $\left.8.94 \mathrm{fm}, c=1.528, a_{s}=1.4\right)$. Although we know that the SS potential does not follow Newton's form [Eq. (19)], we can still fit our $k_{n}$ values by first-order polynomials of the variable $n$ and $\ln (n)$, respectively, to check the validity of Eqs. (20), (23) and (21). Equations (20) and (23) seem to be valid approximately in the $n$ range shown in Fig. 14 for $a_{s} \geqslant 1$. For $\sigma=0.6$, which produced a pocket in Fig. 4, the relation breaks down beyond $n_{s} \approx 12$.

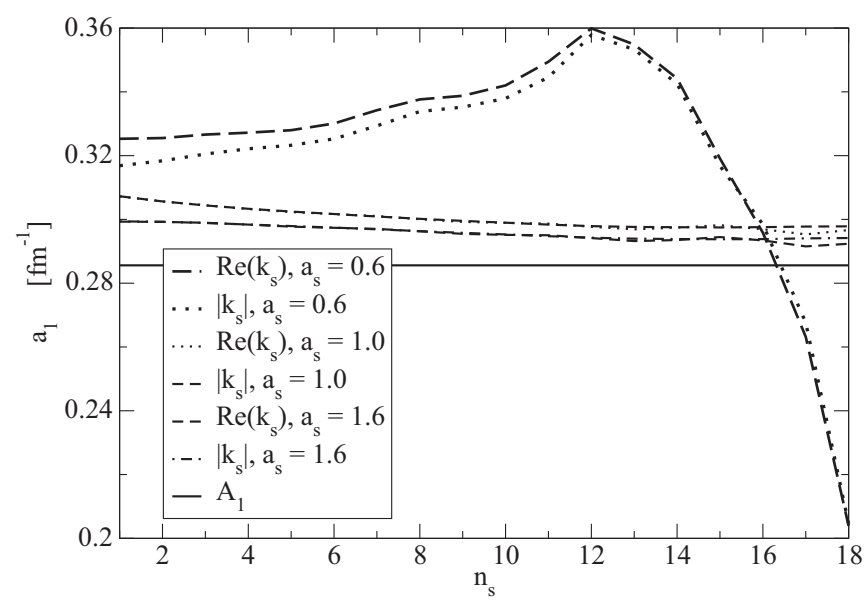

FIG. 14. Slope $a_{1}$ of the straight line fitted to the starting $k_{n}$ values $\left(n_{s}=1, \ldots, n_{u}-1\right.$ and $\left.n_{u}=20\right)$ for $\mathrm{SS}$ potentials of different $a_{s}$, with $a_{s}=1.0$ belonging to the SV potential; $A_{1}=\pi / 11 \mathrm{fm}^{-1}$. Slopes belonging to $a_{s}=1.0$ and $a_{s}=1.6$ are hardly distinguishable in the given scale.
Test calculations show that the $k_{n}^{I}$ values weakly depend on $a_{s}$, and the $\sigma$, defined by Eq. (21), does not seem to converge. That is not surprising as neither the SV nor the SS potential satisfies Eq. (19). Just as for the SV potential, the $k_{n}^{I}$ values show an almost linear slow increase with $n$. This offers practical recipes for finding suitable starting values in searches for $S$-matrix poles.

\section{CONCLUSION}

The conventional nuclear potentials do not tend to zero at finite distances, but are set to zero artificially. Consequently, they have unpleasant mathematical and numerical properties, which cause appreciable errors in broad resonances. Their SFR substitutes have pleasant mathematical and numerical properties, but their tails are unphysical. Here we examined the properties of a family of SFR potentials related to the WS potential, with an emphasis on the effect of the tail and on the pole trajectories belonging to broad resonances.

We concentrated on the SV potential, which consists of a term $\exp \left[\left(r^{2} /\left(r^{2}-\rho_{0}^{2}\right)\right]\left(r<\rho_{0}\right)\right.$ and a term like the derivative of that but with a different parameter $\rho_{1}\left(\leqslant \rho_{0}\right)$. We constructed parameters that fit the real parts of the global Perey-Perey and Becchetti-Greenlees optical potentials best. The best-fit range $\rho_{0}$ of the SV potential is found to scale by $A_{T}^{1 / 3}$ for both geometries, and the difference of the two ranges, $\rho_{0}-\rho_{1}$, is positive and it is three to four times of the diffuseness of the WS potential. The admixture of the derivative term tends to zero with decreasing mass number.

In fact, it was found that, for light nuclei, the phenomenological neutron potential can be approximated reasonably well by a single-term SV potential, and the single-particle energies and densities calculated in the cutoff WS potential are also reproduced. In this case the form factor of the potential has a single parameter, its range $\rho_{0}$. The tail of the density is pretty reasonable because it is determined primarily by the energies, and those are reproduced well by the SV potential.

The new potential form (SS) introduced by Sahu and Sahu [2] can be considered as a generalization of the SV form. The extra diffuseness parameter may smooth or roughen the potential in the region around $\rho_{1}$ depending on whether $a_{s}>1$ or $a_{s}<1$.

The range of the SFR potentials determines approximately the starting points of the pole trajectories belonging to potential strength zero. The problem of the $S$-matrix poles becomes ill defined in a potential with strength $V_{0} \approx 0$; thus, it is important to see whether the computer code is able to solve the problem for small $V_{0}$. A check is provided by potentials of the form of $-V_{0}(R-r)(r \leqslant R)$, for which these starting points are approximately determined apart from an additive constant. This check has shown that our calculations are remarkably accurate.

It is more surprising that even though the CWS and the SV potentials are very different in the neighborhood of the cutoff, the pole trajectories of the SV potentials bear out some of the properties of those of the $-V_{0}(R-r)$ potentials, especially for large node numbers. For some low values of the node number, the CWS trajectory shows strange shapes, while the SS and SV potentials behave absolutely regularly. The pole 
trajectories of the SS potential depend weakly on the extra diffuseness parameter.

In conclusion, the present results are reassuring concerning the use of the SFR potentials. The starting points of the pole trajectories seem to have some approximate universality properties, which can be used to estimate the values of these starting points.

\section{ACKNOWLEDGMENTS}

This work was partially supported by the ENIAC CSI No. 120209 project and by the TÁMOP-4.2.2.C-11/1/KONV2012-0001 project. The latter project has been supported by the European Union, cofinanced by the European Social Fund.
[1] P. Salamon and T. Vertse, Phys. Rev. C 77, 037302 (2008).

[2] B. Sahu and B. Sahu, Int. J. Mod. Phys. E 21, 1250067 (2012).

[3] A. Rácz, P. Salamon, and T. Vertse, Phys. Rev. C 84, 037602 (2011).

[4] J. Darai, A. Rácz, P. Salamon, and R. G. Lovas, Phys. Rev. C 86, 014314 (2012).

[5] H. Bremmermann, Distributions, Complex Variables, and Fourier Transform (Addison-Wesley, Reading, MA, 1965), p. 13.

[6] I. Nándori, J. High Energy Phys. 04 (2013) 150; I. Nándori, I. G. Márián, and V. Bacsó, Phys. Rev. D 89, 047701 (2014); I. G. Márián, U. D. Jentschura, and I. Nándori, J. Phys. G 41, 055001 (2014).

[7] F. G. Perey, Phys. Rev. 131, 745 (1963).
[8] F. D. Becchetti and G. W. Greenlees, Phys. Rev. 182, 1190 (1969).

[9] X.-H. Li and L.-W. Chen, Nucl. Phys. A 874, 62 (2012).

[10] P. Mohr, G. G. Kiss, Zs. Fülöp, D. Galaviz, Gy. Gyürky, and E. Somorjai, At. Data Nucl. Data 99, 651 (2013).

[11] D. S. Delion and R. J. Liotta, Phys. Rev. C 87, 041302(R) (2013).

[12] H. M. Nussenzveig, Nucl. Phys. 11, 499 (1959).

[13] R. G. Newton, Scattering Theory of Waves and Particles (Springer, New York, 1982).

[14] T. Regge, Il Nuovo Cim. 8, 671 (1958).

[15] L. Gr. Ixaru, Numerical Methods for Differential Equations and Applications (D. Reidel, Dordrecht, 1984).

[16] L. Gr. Ixaru, M. Rizea, and T. Vertse, Comput. Phys. Commun. 85, 217 (1995). 